\title{
Growth and form of two-dimensional rotating aggregates
}

\author{
N. Lemke, M. G. Malcum, R. M. C. de Almeida, P. M. Mors, and J. R. Iglesias \\ Instituto de Física, Universidade Federal do Rio Grande do Sul, Caixa Postal 15051, 91501-970 Porto Alegre, \\ Rio Grande do Sul, Brazil
}

(Received 6 October 1992)

\begin{abstract}
We propose a two-dimensional particle-cluster aggregation model which considers explicitly linear and angular momentum conservation. Each aggregate is grown from a seed of given mass and given initial angular velocity, to which particles of unitary mass and ballistic trajectories are added. The fractal dimensions of the resulting aggregates are calculated and Fourier transform analysis is performed. Very different features are found, as initial conditions are varied. Particularly, spiral structures are found for a seed of high mass and high initial angular velocity.
\end{abstract}

PACS number(s): 05.40.+j, 81.35.+k, 95.30.-k

\section{INTRODUCTION}

Shape and growth of natural systems are definitely influenced by the conditions imposed by conservation laws of physical quantities. Particularly, conservation of angular momentum and the consequent rotation play an essential role in spiral structures as vortices or galaxies. Evidently, specific details are relevant and may not be discarded when the purpose is to obtain quantitative predictions about measurable characteristics of a given system. However, when different systems may present very similar patterns, one can guess that some universal law should be determinant. For example, the overall pattern in cellular structures such as soap bubbles, polycrystalline grains, and biological tissues are determined by the maximum entropy, subjected to topologic and geometric constraints [1].

Aggregation models provide a useful technique to investigate the role played by different conditions on the growth dynamics of a system. It is an interesting point to apply such a technique to investigate the influence of rotation on the final shape of a cluster. Rotating aggregates in the context of cluster-cluster aggregation have been previously studied [2], but not fully considering the classical mechanics conservation laws.

Here we propose a two-dimensional aggregation model where conservation of total angular and linear momenta are explicitly considered. A system made of many aggregated particles may store angular momentum in two channels: the motion of its center of mass and the relative motion of its particles. Hence, when a new particle is aggregated to the system, through an inelastic collision, its angular momentum is distributed in these two channels and both center of mass velocity and angular velocity are changed in order to keep constant total linear and angular momenta.

We consider an initial seed, with given mass $m_{0}$, unitary radius, and initial angular momentum $L_{0}$, and incident particles with unitary mass following ballistic trajectories coming from all directions with random velocities.
In the absence of rotation one recovers a ballistic version of the Witten-Sander model $[3,4]$.

To investigate the morphology of different aggregates we determine a fractal dimension and perform a twodimensional (2D) Fourier analysis of their particle distributions, taking as basis functions logarithmic spirals, which are specially adequate to study spiral point distributions [5]. A crossover from a Witten-Sander-like behavior to a structure which presents a unique logarithm spiral arm is observed.

The paper is organized as follows: Sec. II introduces the model; in Sec. III we present the calculation procedure to obtain the aggregate fractal dimensions and discuss the spiral transform; Sec. IV presents and discusses the results; and, finally, in Sec. V we summarize our conclusions.

\section{MODEL}

We consider $2 \mathrm{D}$ off-lattice, particle-cluster aggregation $[3,4]$, with the incident particles describing straight trajectories. The simulation begins with an initial seed of unitary diameter, mass $\mathrm{m}_{0}$, and angular velocity $\omega_{0}$, lying at $x=0, y=0$. Particles with unitary mass and unitary diameter are thrown towards the seed with random velocities from random points of a circle centered at the center of mass of the growing cluster, of radius $1.5 r_{\max }$, where $r_{\max }$ is the maximum radius of the cluster. When an incident particle touches the cluster it sticks to it irreversibly. Particles that do not touch the cluster just leave the circle. The process is repeated until the final aggregate is obtained.

Total linear and angular momenta are conserved, i.e., cluster linear and angular momenta are "updated" by adding the momenta transferred by the incoming particle.

Particle speed is a random variable uniformly distributed over the interval $[10 d / s, 50 d / s]$, where $d$ is the particles diameter and $s$ is one "simulation time step." 
This ensures a range of possible values for the particle's probability of capture by the growing cluster. The velocity direction is also randomly distributed.

The simulations were performed on a Sun Sparcstation 2 workstation, and five aggregates of about $10^{4}$ particles each have been constructed for each set of initial conditions: initial mass $\mathrm{m}_{0}$ equal to 1 , $10^{6}$, and $10^{12}$ (where the incident particles have unitary mass) and initial angular velocities $\omega_{0}$ equal to $0,0.1,0.2,0.3,0.4,0.5,1.0,1.5,2.0,2.5$, and $3.0 \mathrm{rad} / \mathrm{s}$.

Typical aggregates are shown in Fig. 1. In the case of unitary initial mass, one recovers a ballistic WittenSander aggregation because after a very short transient the angular velocity of the aggregate with respect to its center of mass decays to zero (due to the increase in the moment of inertia of the cluster). For $\mathrm{m}_{0}=10^{6}$ interesting behaviors appear, the angular velocity decays slowly, and many particles may be added to the cluster before it stops rotating. The "infinite initial mass" case is attained when the initial mass is large enough to guarantee that angular velocity remains constant during the aggregation process. Here, constant rotation occurred with $m_{0}=10^{12}$; however, if enough time is allowed, enough mass will be added to the cluster and its angular velocity will finally decay to zero. We shall concentrate on the $m_{0}=10^{6}$ and $10^{12}$ aggregates, where interesting features can be detected.

\section{CLUSTER CHARACTERIZATION}

Different fractal dimensions $[4,6]$ are typical parameters used to characterize the final products of aggregation simulations. However, this characterization is very poor to investigate some specific morphologic features that are apparent in aggregates as those shown on Fig. 1: very different aggregates may have the same fractal dimension. Hence, a further characterization is required and we shall use a two-dimensional transform over the set of points associated with the positions of the particles in the cluster. This is a 2D Fourier transform with the basis functions being logarithmic spirals, which are especially adequate to put in evidence spiral arm structure that a set of points may present [7-9].

\section{A. Box-counting dimension}

Consider $M$, a set of points in a plane whose fractal dimension is to be calculated. Consider also a square lattice with lattice parameter $\delta$ over the set. Box-counting dimension $D_{B}$ is defined as the limit (whenever it exists)

$$
D_{B}=-\lim _{\delta \rightarrow 0} \frac{\ln N_{\delta}}{\ln \delta}
$$

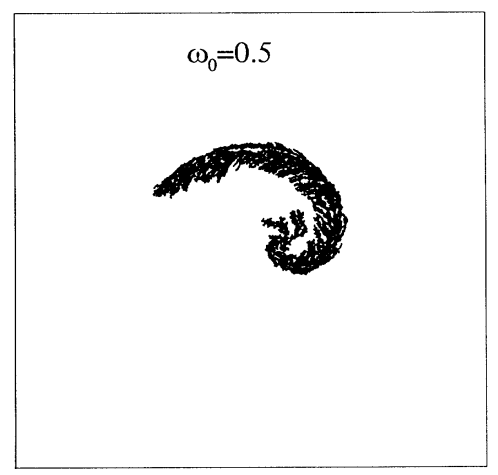

(a)

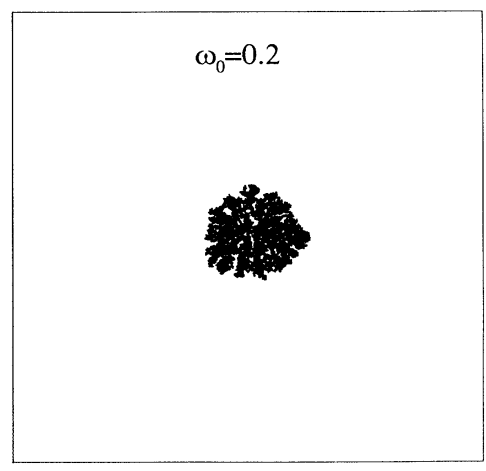

(d)

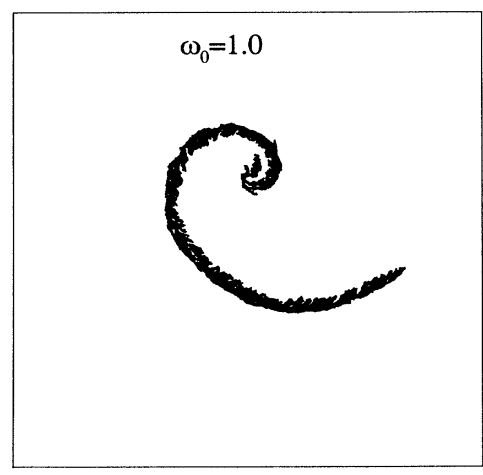

(b)

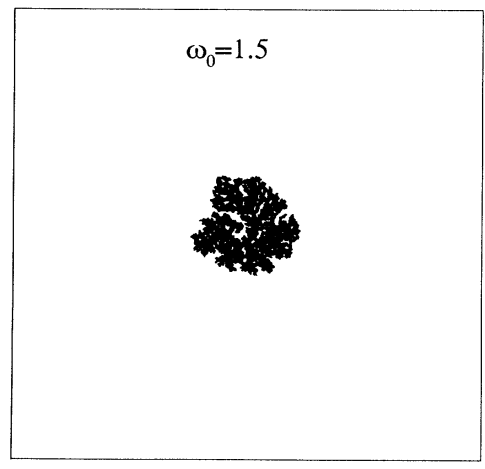

(e)

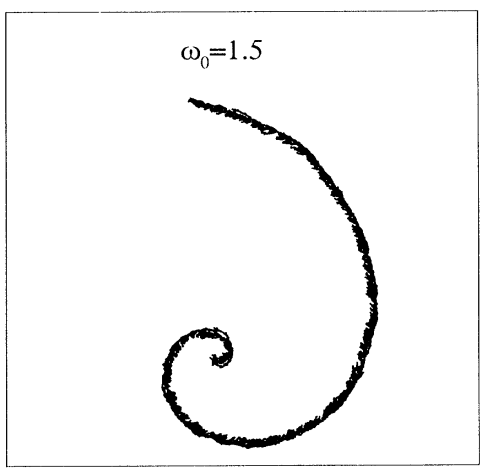

(c)

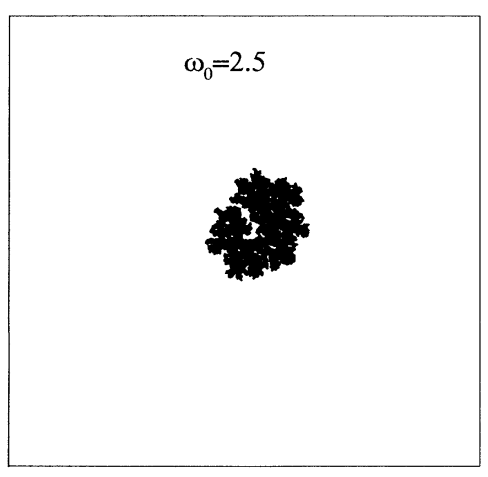

(f)

FIG. 1. Typical clusters obtained from simulations. (a)-(c) The infinite mass regime is obtained for $\mathrm{m}_{0}=10^{12}$ and (d)-(f) the finite-mass regime for $\mathrm{m}_{0}=10^{6}$. The aggregates have the following fractal dimensions: (a) 1.75 , (b) 1.69, (c) 1.58 , (d) 1.84 , (e) 1.83 , and (f) 1.82 . 
where $N_{\delta}$ is the number of cells containing at least one point of the set [10].

We averaged $D_{B}$ for five different aggregates with the same initial conditions. Care was taken with lower and upper cutoffs, defined, respectively, by the diameter of the aggregated particles and the size of the aggregate. The number of particles in the aggregates is typically $10^{4}$. The ballistic Witten-Sander model builds up compact cluster, but this result is numerically very difficult to obtain $[11,12]$ : simulations up to 25000 particles were necessary to reach a value near 2 for fractal dimension, in the $2 \mathrm{D}$ case. The reason for this is the number of large holes that are also "grown" with the aggregate. A similar effect may also be present in our aggregation and finitesize effects must be taken into account, as discussed in Sec. IV.

\section{B. 2D Fourier transform}

Here we consider a Fourier transform which was first introduced to study spiral galaxies $[5,7-9]$ and is especially adequate to study the morphology of spiral clusters.

Consider a function $f(r, \theta)$, defined in the real plane in polar coordinates. The Fourier transform $E_{n}(p)\{f\}$ of the function $f$ is defined as [9]

$$
E_{n}(p)\{f\}=\int_{-\infty}^{\infty} \int_{-\pi}^{\pi} f(r, \theta) e^{i(p \ln r+n \theta)} d(\ln r) d \theta
$$

where $p$ is real and $n$ is an integer. $E_{n}(p)$ is complex and it is convenient to define a real quantity $I_{n}(p)$ as

$$
I_{n}(p)=\left|E_{n}(p)\right|^{2} .
$$

As $n$ is a discrete parameter, this Fourier transform yields a collection of spectra $I_{n}(p)$. To verify the adequacy of this transform in analyzing spiral aggregates, consider the following function $f(r, \theta)$ that represents $N$ points distributed over the real plane

$$
f(r, \theta)=\frac{1}{N} \sum_{j=1}^{N} \delta\left(r-r_{j}\right) \delta\left(\theta-\theta_{j}\right)
$$

where $\left(r_{j}, \theta_{j}\right)$ are the polar coordinates of the $j$ th point. The resulting spectrum for this distribution is given by

$$
I_{n}(p)=\left|\frac{1}{N} \sum_{j=1}^{N} e^{i\left(p \ln r_{j}+n \theta_{j}\right)}\right|^{2}
$$

where the factor $N$ is introduced for normalization purposes.

Pronounced peaks are found around $p=-n / \alpha$ whenever the points are distributed along a single logarithmic spiral given by $r=\exp \alpha \theta$, where $\alpha$ is the tangent of the pitch angle, that is, the angle between the perpendicular to the position vector and the tangent vector at any point belonging to the spiral. In the case of points distributed over two spirals with the same pitch angle but with a phase difference of $\pi$ between the arms, peaks will
TABLE I. Relation of geometric aspects of the spirals with the spectra.

\begin{tabular}{ll}
\hline \hline $\begin{array}{l}\text { Geometric } \\
\text { characteristics }\end{array}$ & $\begin{array}{l}\text { Effects on } \\
\text { spectra }\end{array}$ \\
\hline $\begin{array}{l}\text { Number of spiral arms } N \\
\text { Pitch angle } \alpha\end{array}$ & $\begin{array}{l}\text { Peaks when } m=k N \\
\text { Position of the peak }\end{array}$ \\
Arm thickness $\sigma$ & $\begin{array}{l}\text { Peak intensity } \\
\text { Arm's length }\end{array}$ \\
\hline \hline
\end{tabular}

be found only for even $n$. Analogously, when the points are distributed over $k$ logarithmic spirals with the same pitch angle and with phase differences of $(2 \pi) / k$ between each arm, peaks will only be found for values of $n$ that are multiples of $k$.

It is clear that the set of points we are interested in analyzing is finite and the points are not exactly distributed over logarithmic spirals. The arms are also finite and have finite width. Nevertheless, the pitch angles are reasonably constant for each aggregate and the phase difference are roughly $(2 \pi) / k$ for $k$-armed structures.

The consequence of the width and finite length of the aggregate arms, together with statistical variations of the pitch angle, is to make the analysis of resulting spectra more complex: peaks may overlap and/or grow wider and less intense. Table I summarizes this scenario.

Here we follow the protocol suggested by Refs. [5,7-9] for the analysis of the spectra of the aggregates: we investigate the values of $n$ where there are peaks, and compare their intensity and width. The results are presented in the following sections, together with the measures of the fractal dimension $D_{B}$.

\section{RESULTS}

Three situations are considered.

(i) Unitary initial mass, when the angular velocity rapidly decays to zero. In this case, after few simulation steps, the aggregate is equivalent to the Witten-Sander ballistic model, and no new interesting phenomena were detected.

(ii) Finite initial mass, typically $m_{0}=10^{6}$. Here the angular velocity decays slowly. The inner core structure is then highly influenced by the angular velocity but the external structure may evolve to a circularly symmetric one, depending on the initial values of the angular momentum. In this case one or more arms may appear. It will be discussed in detail in Sec. IV A.

(iii) Infinite initial mass, typically $m_{0} \geq 10^{12}$, where the angular velocity remains approximately constant. Very different final patterns may appear, depending on the initial angular velocity. This case also deserves special attention and we shall return to it in Sec. IVB.

\section{A. Finite initial mass}

With $\mathrm{m}_{0}=10^{6}$ the increase in the moment of inertia is sufficient to decrease the angular velocity, but the 
decay is slow enough to allow many particles to be aggregated before it goes to zero. As a consequence, the inner structure of the aggregate may have a different morphology from its outer parts. When the aggregate rotates with a non-negligible angular velocity its outer extremities screen the interior and one or more spiral arms may grow. On the other hand, as particles are aggregated, the moment of inertia increases, the angular velocity decreases and the screening of inner parts become less efficient: the arms tend to widen up until the angular velocity is practically zero and there is a circularly symmet-

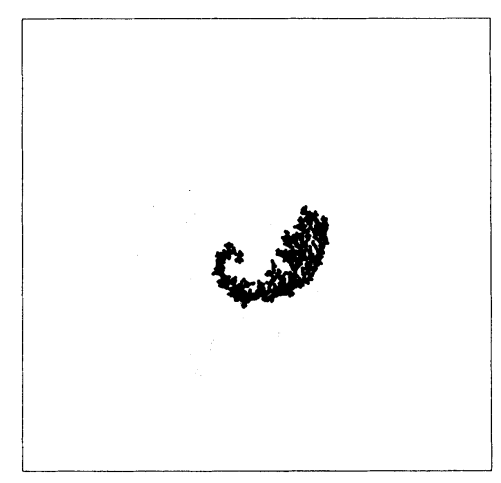

(a)

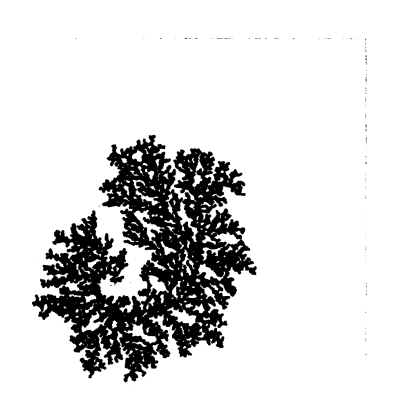

(b)

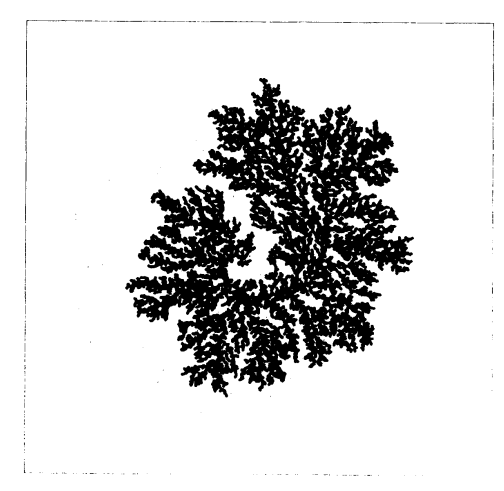

(c)

FIG. 2. Three snapshots of a cluster with $m_{0}=10^{6}$ and $\omega_{0}=2.5$ for (a) 1000 , (b) 6000 , and (c) 9920 aggregated particles. The broadening of this spiral arm causes the decrease of the Fourier-transform peak intensity in Fig. 3.

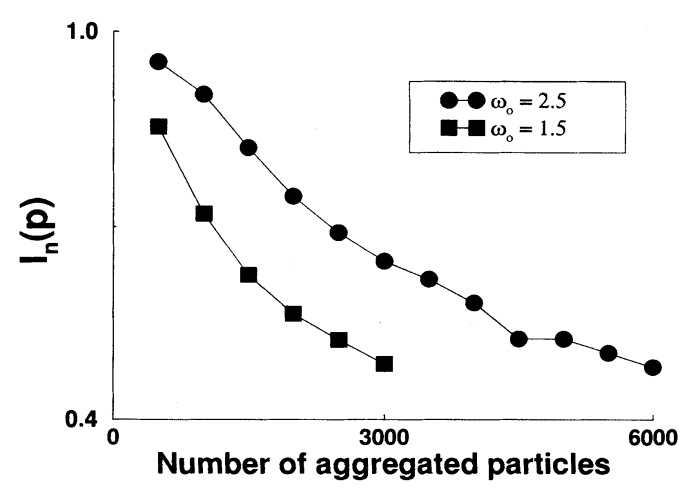

FIG. 3. Dependence of the strongest peak intensity for $n=1$ on the number of aggregated particles for clusters with $m_{0}=10^{6}, \omega_{0}=1.5$ and 2.5 .

ric probability of aggregating particles for every exterior point of the clusters, destroying spiral distributions.

The greater the initial angular velocity, the faster the increase in the moment of inertia, due to more efficient screening and, consequently, to larger distances from the center of mass to the sticking points where new particles are aggregated: the higher rate to increase the moment of inertia compensates the higher initial angular velocity. In this case of finite initial mass, the aggregates present approximately the same form and, consequently, the same box-counting dimension for all initial angular velocities, roughly 1.8 (see Fig. 1).

Figure 2 shows three snapshots of a finite initial mass cluster growth, at different stages. The initial growth of spiral arms and the posterior broadening of the arms are clearly displayed.

The slowing down of the angular velocity and its consequences in the structure of the cluster are also detected by the Fourier analysis. Figure 3 shows the most intense peak $I_{n}(p)$ for the aggregate shown in Fig. 2: the intensity decreases as the number of particles grows, due to the broadening of spiral arms.

\section{B. Infinite initial mass}

We consider infinite initial mass when the initial moment of inertia is much larger than the moment of inertia added to the cluster by all aggregated particles. This limit is attained in the simulations for $m_{0}=10^{12}$, and yields a constant angular velocity throughout the growth process. While for a finite mass the interesting feature is the crossover from a regime with a finite initial angular velocity to a zero one, here the interest is exactly the opposite. As the patterns are determined by a constant value of angular velocity, this case is especially suitable for an investigation of the effects of rotation in the final form of the aggregates. We investigate the dependence of the structure of the cluster with angular velocity. For $\omega_{0}=0$, the ballistic Witten-Sander model, $D_{B}=2$, is not yet attained due to finite-size effects which are still important for $10^{4}$ particles, as shown in Fig. 4. 


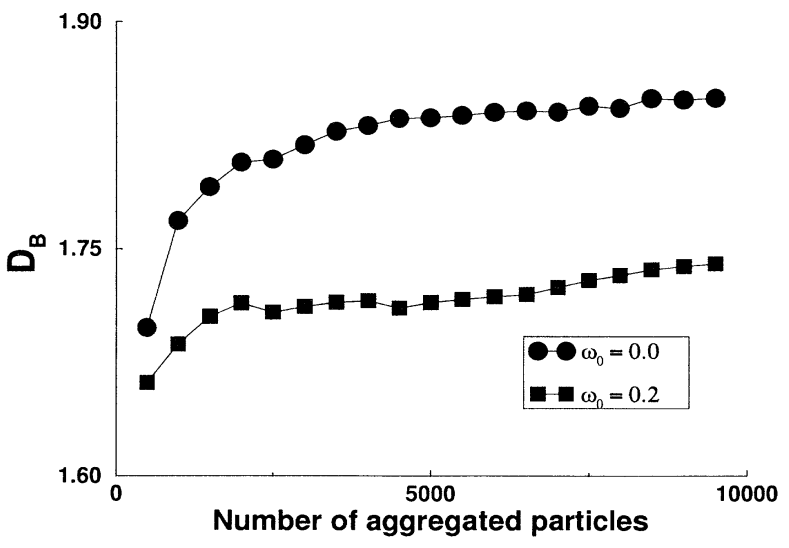

FIG. 4. Plot of $D_{B}$ against the number of aggregated particles for $\omega_{0}=0.0$ and 0.2 in the infinite initial mass limit.

The dependence of $D_{B}$ with $\omega_{0}$ in this case is shown in Fig. 5. The behavior is not trivial: there is a minimum at $\omega_{0}=0.2$. This effect is a consequence of different screening regime of the inner core. Figure 6 shows three different aggregates, for $\omega_{0}=0,0.2$, and 0.5 (before, at, and after the minimum).

For $\omega_{0}=0.2$ one observes formation of many spiral arms. These arms, although not sufficiently defined to present very peaked structures in its spiral transform spectra, are complex enough to screen some inner parts and to lower the cluster fractal dimension.

For $\omega_{0}=0.5$ the inner complex structure is completely screened by the longest arm: the angular velocity becomes high enough to strongly favor sticking at the outer points. More exterior points means more efficient screening: as the largest arm grows, the structure tends to present a unique spiral arm. The Fourier analysis clearly detects the crossover, which can be described as a process that begins with a circularly symmetric structure and evolves towards a unique spiral arm, passing through stages of many spiral arms which are successively screened by the largest one. Figure 7 shows the

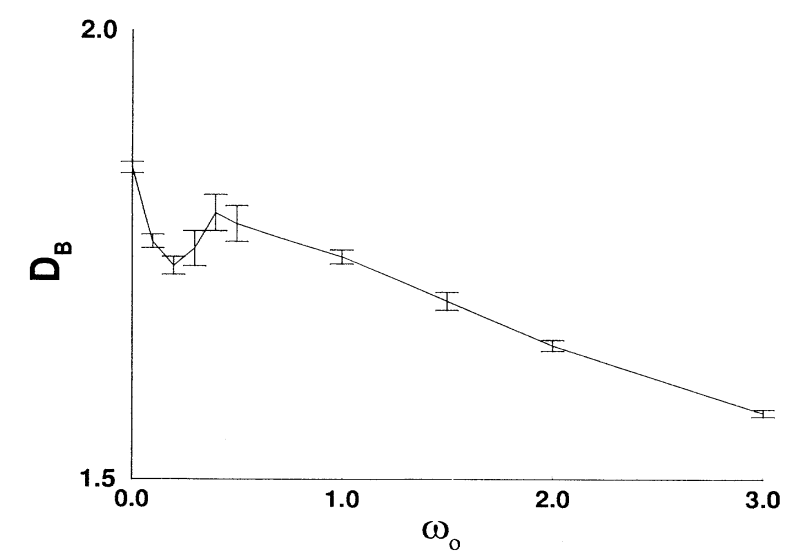

FIG. 5. Dependence of $D_{B}$ with $\omega_{0}$ for $m_{0}=10^{12}$. The averages are taken over five aggregates. spectra for this case. The relative intensities of $n=1$ and $n=2$ peaks signals the crossover from a two arm structure [where $\mathrm{I}_{2}(p)>\mathrm{I}_{1}(p)$ ] to a unique arm cluster $\left[\mathrm{I}_{1}(p)>\mathrm{I}_{2}(p)\right]$. This figure also presents the same plots for $m_{0}=10^{12}$ and $\omega_{0}=1.5$, where there is no crossover because at this angular velocity we can only detect a unique arm structure evolving since the beginning of the aggregation process.

Figure 8 shows the intensity of the most intense peak against $n$ for different values of $\omega_{0}$ : as the angular velocity increases, the structure tends to present an unique

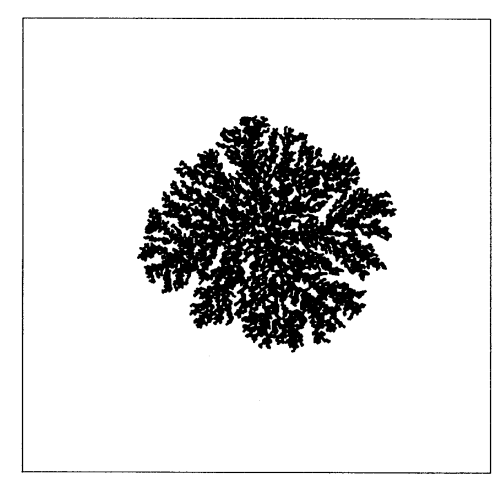

(a)

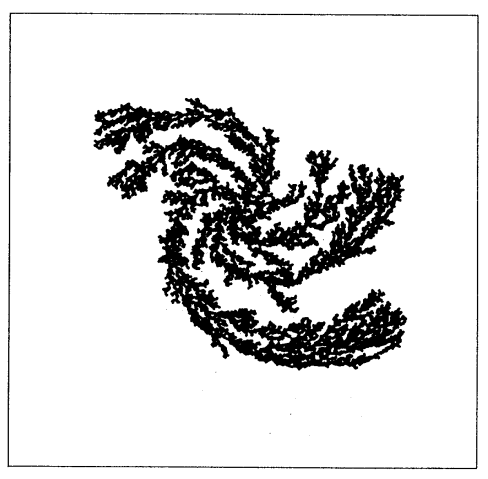

(b)

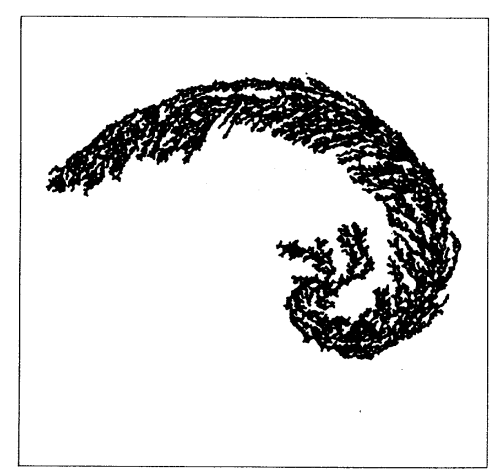

(c)

FIG. 6. Three clusters with $m_{0}=10^{12}$ and (a) $\omega_{0}=0$, (b) $\omega_{0}=0.2$, and (c) $\omega_{0}=0.5$. These aggregates correspond to the points before, on, and after the minimum of Fig. 5 . 


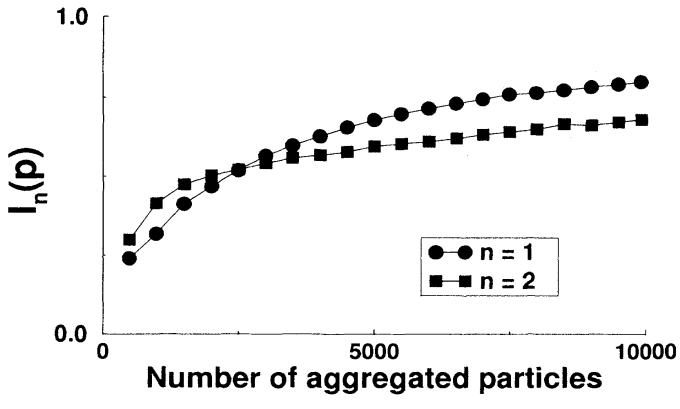

(a)

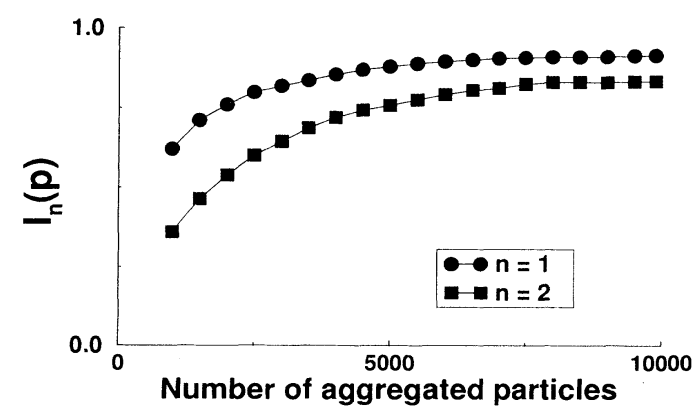

(b)

FIG. 7. Intensity of the stronger peak for $n=1$ and 2 in the infinite mass regime for (a) $\omega_{0}=0.5$ and (b) $\omega_{0}=1.0$.

arm, progressively better defined, with a sharply peaked spectrum $I_{1}(p)$.

In summary in the infinite initial mass case, two different behaviors emerged, determined by the initial angular velocity. For $\omega_{0}<0.2$, the patterns are very complex, many spiral (proto) arms may evolve, the fractal dimension decreases from 1.8 to 1.5 with increasing $\omega_{0}$ and the spectra $I_{n}(p)$ is very complex but qualitatively equivalent to those found for some spiral galaxies $[7,8]$, reflecting the

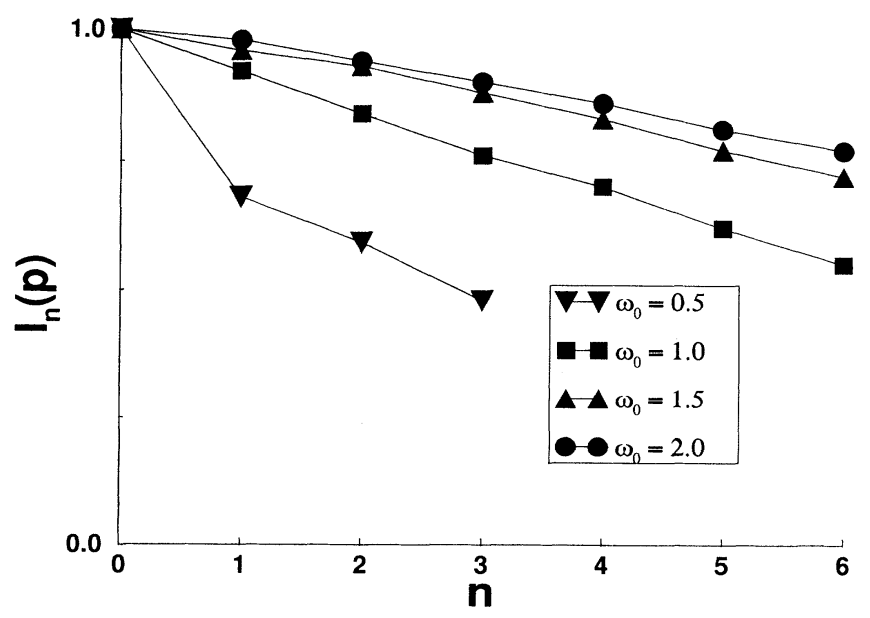

FIG. 8. Intensity of the most intense peak against $n$ for $\omega_{0}$ $=0.5,1.0,1.5$, and 2.0 and $m_{0}=10^{12}$. similarity of these aggregates with that structures. For $\omega_{0}>0.2$, on the other hand, at the beginning of the aggregation process the structure starts presenting many arms, but as more particles are aggregated, the longest arm screens the other ones and the external structure tends to a unique spiral arm pattern. The rate at which this larger arm effectively screens the internal structure depends on the angular velocity. For low values, near 0.2 , this happens much slower and a complex inner structure may grow before it is completely screened by the external arm while for high values of angular velocity there is no internal structure because a unique arm is favored from the very beginning of the process (see Fig. 1). This dynamics explains the plots in Fig. 5: besides the broadening of the larger arm due to a lower angular velocity, the complex internal structure also decreases the fractal dimension for low values of $\omega_{0}$.

\section{CONCLUSIONS}

Here we presented an off-lattice, particle-cluster aggregation model, where the angular and linear momenta are explicitly conserved, by considering inelastic collisions of the particles with the cluster. We varied the initial seed mass and angular momentum. The resulting aggregates present very different morphologies, which have been analyzed through the determination of the box-counting dimension and 2D Fourier-transform spectra. This last technique revealed itself especially suitable to study the morphology of rotating aggregates because the aggregates may present spiral arms.

Three initial mass conditions have been considered: unitary mass, which stops rotating rapidly; finite mass, where the angular velocity varies from an initial value down to zero during the aggregation process; and infinite mass, where the angular velocity remains constant throughout the simulation. The second and third cases exhibit different patterns and interesting features.

For initial finite mass, the final aggregate form is very similar and qualitatively independent of the initial angular velocity. There is an internal structure, where many (proto) spiral arms may appear, but as the aggregate stops rotating, the arms broaden up and the outer structure tends to get circularly symmetric. No peaked spectra emerges from the spiral transform analysis.

In the case of infinite initial mass, the final form of the aggregate strongly depends on the initial angular velocity. For low angular velocities, a complex structure emerges, with many broad spiral arms similar to the structure of spiral galaxies. The fractal dimension $\left(D_{B}\right)$ decreases with increasing angular velocity up to $\omega_{0}=0.2$, where it reaches a minimum. At this angular velocity, as more particles are aggregated, the longest arm screens the others, and a external, single-armed structure screens a complex inner core. The fractal dimension increases a little with angular velocity, and decreases again, because a too large angular velocity causes the width of the spiral arm to shrink. When the angular velocity becomes too large no internal structure is formed and, in the $\omega_{0} \rightarrow \infty$ limit, the cluster tends to a one- 
dimensional object. The 2D Fourier-transform spectra manages to detect and quantify all these different behaviors and crossovers.

On the other hand, when enough time is allowed, the "infinite initial mass" aggregates should stop rotating and present a circularly symmetric external structure envolving an internal complex core, as in the finite mass regime. The size and the complexity of this inner core will be extended, and may deserve a careful investigation, but due to the computation time required it is beyond the aim of this paper.

Many physical systems may present spiral patterns. In particular, spiral galaxies are found to present from two to many spiral arms, very tightly wound or loosely open, narrowly or widely spread along a (logarithmic) spiral direction (for a review see Ref. [13] and references therein). Their 2D Fourier spectra show that few components provide a good representation of their shape (ionized hydrogen region distributions) [7-9]. The logarithmic spiral character also appears in our aggregates, and can be taken as an indication of the role that angular momentum conservation and the consequent rotation movement plays in the growth of spiral arms in galaxies. A systematic investigation of these natural systems through aggregation models that conserve total angular momentum may bring some further insight about the physics governing the dynamics of growth of spiral arms in galaxies.

\section{ACKNOWLEDGMENTS}

We acknowledge L.G. Brunnet, J.M. Mombach, and J.J. Arenzon for interesting suggestions. We especially thank H. Dottori and I. Puerari for the 2D Fourier transform discussions. This work was partially supported by Brazilian agencies CNPq (Conselho Nacional de Desenvolvimento Científico e Tecnológico), FAPERGS (Fundação de Amparo à Pesquisa do Estado do Rio Grande do Sul), and FINEP (Financiadora de Estudos e Projetos).
[1] J.R. Iglesias and R.M.C. de Almeida, Phys. Rev. A 43, 2763 (1991).

[2] P. Meakin, J. Chem. Phys. 81, 4637 (1984).

[3] T. Witten and L. Sander, Phys. Rev. Lett. 47, 1400 (1981).

[4] R. Jullien and R. Botet, Aggregation and Fractal Aggregates (World Scientific, Singapore, 1986).

[5] A.J. Kalnajs, Astrophys. J. 166, 275 (1975).

[6] B. Mandelbrot, The Fractal Geometry of Nature (Freeman, New York, 1982).

[7] S. Considère and E. Athanassoula, Astron. Astrophys.
111, 28 (1982).

[8] S. Considère and E. Athanassoula, Astron. Astrophys. Suppl. Ser. 76, 365 (1988).

[9] I. Puerari and H.A. Dottori, Astron. Astrophys. Suppl. Ser. 93, 469 (1992).

[10] K.J. Falconer, Fractal Geometry (Wiley, West Sussex, 1990).

[11] D. Bensimon, E. Domany, and E. Aharony, Phys. Rev. Lett. 51, 415 (1983).

[12] P. Meakin, J. Colloid Interface Sci. 16, 415 (1983).

[13] P. E. Seiden and L. S. Schulman, Adv. Phys. 39, 1 (1990). 